\title{
How Combining Grounding and Acupuncture Simultaneously Increases Beneficial Outcomes Over Traditional Acupuncture?
}

\author{
Madiha Khalid and Jeremy Madvin
}

\section{ABSTRACT}

\begin{abstract}
Multidisciplinary studies showed that when the human body is electrically in contact with the Earth (earthing or grounding), it produces intriguing consequences on the physiology and health of the human being. These effects are related to treating acute and chronic inflammatory and autoimmune illnesses, immunological responses, wound treatment, and prevention. At the same time, acupuncture in Traditional Medicine has been widely utilized for its low side effects and therapeutic effectiveness as primary therapeutic therapy. However, the electrical reaction to acupuncture stimulation has not been investigated in conditions with acupuncture that could alter acupuncture therapeutic effectiveness. The study's objective is to inform scientists of the apparent innovative approach to inflammation studies; and alert researchers to the importance of the duration and the degree of grounding, which may affect the results of inflammation, injury, and tumorigenesis studies. Grounding with acupuncture decreases pain and changes the quantity of circulating neutrophils and lymphocytes and circulating chemical substances linked to inflammation. This work reviews the potential of acupuncture with earthing as an easy and readily accessible worldwide modality of substantial clinical importance is reviewed in this work.
\end{abstract}

Keywords: Acupuncture Earthing, grounding, inflammation, pain.
Submitted: August 23, 2021

Published: September 23, 2021

ISSN: 2593-8339

DOI: $10.24018 /$ ejmed.2021.3.5.1025

\section{Khalid *}

Humdard University, Islamabad Campus, Pakistan.

(e-mail: msmadihakhalid@gmail.com)

J. Madvin

MBA International Business

The University of Redlands, United

States.

(e-mail:jeremym@zetatherapy.com)

*Corresponding Author

\section{INTRODUCTION}

External physical stimulations trigger chemical and electrical reactions that influence vital biological functions in humans. Acupuncture is also thought to elicit such responses in Traditional medicine, helping unbalanced physiological states return to normal healthy physiological conditions. The physiological philosophy of acupuncture has been mentioned in Western medicine as a nerve and endocrine cell regulation approach based on cytology [1]. The following research confirms the principles of acupuncture: the effect of acupuncture anesthesia is transmitted by cerebrospinal fluid [2], and the sensory system is stimulated by complex neural nerve or sensory receptor [3]. Cho et al. [4] study were noteworthy because it revealed the acupuncture principle. Even though acupuncture stimuli cause the brain to act, it's probable that not all acupuncture effects are transmitted to the nervous system because the physiological response to external stimuli is considerably faster than the brain can process [5]. It indicates that acupuncture treatments may also include biological processes besides the nervous system. Therefore, acupuncture involves the transfer of electrical energy via nerve or connective tissue [6].

Acupuncture stimulation has been shown to produce an ion-electric charge [7], which is then conveyed through several biological routes [8]. Furthermore, it is well understood that acupuncture therapy's effectiveness depends on the number of electric charges and the frequency of acupuncture stimulus [9]. Acupuncture activation of the Meridian cycle amplifies small currents and voltages produced by this circuit [10]. This electricity could be induced by the electric potential created by the current generated by the activation of acupuncture points with an acupuncture needle or from the chemical reaction between the acupuncture needle and the hypodermis [11]. It is because chemical reactions alter the ion distribution and bioelectrical charge the same way as electric responses.

Meridians can be thought of as ion-electric-charge channels. Unbalanced positively and negatively charges are the outcome of blocking the electric current of ions in a meridian. It causes pain and the occurrence of various diseases. To restore normal health, acupuncture modifies the elevated or deficient situation of the ion-electric charge. It suggests that acupuncture has a close relationship with the transfer of bioelectrical charges. But acupuncture dependent on the principle of the meridians does not always produce positive therapeutic outcomes, and even when exactly similar methods are used, the results can be quite varied. 
Acupuncture technologies have been implemented for a variety of therapeutic uses. However, existing research does not provide quantitative methods for objectively assessing the effect of acupuncture.

Due to its persistent low effects even under similar experimental conditions, acupuncture currently has limited importance and reliability as a therapy technique. The goal of this study was to objectively explore the impacts of electric grounding and insulation settings in acupuncture stimulation to address this problem substantially.

\section{Why Do We Stimulate Acupressure With GROUNDING?}

Every living creature has electrical charges within its body, according to modern science. It's no different with the human body. We need the ground to keep electrical stability-after all, we are electric beings-and too important for human circadian rhythm, which guides the wide variety of biological processes our bodies regularly perform.

Dr. Reinhold Voll of Germany used an electrical probe to analyze electrical resistance at various places on the body. He discovered multiple body points that yielded unexpected readings, had lower electric resistance, and were distributed along defined routes [12]. Nakatani, a Japanese doctor, have used an electrical device to examine his patients at the time. Nakatani also discovered several low electrical resistance areas that he linked to routes. The meridian of traditional medicine is nearly analogous to these electrical circuits. In addition, the points are identical to acupuncture points in terms of their electrical resistance. Chi refers to the flow of electrical current using low-resistance pathways. The digestive, cardiovascular, neurological, and reproductive systems are all part of the higher organism. Meridians situated under the epidermis and linked to the major organs may be another system associated with low electrical resistance routes [13]. Acupoints are analogous to boost stations in the meridians that send DC signals by connecting acupuncture points to these meridian lines. Different electrical properties exist at acupoints along meridians, and these qualities are employed for diagnostic purposes. When a tissue or organ is fit and active, acupoints show almost no electrical activity [14]. A current is formed locally at the site of damage if injury or infection occurs [15]. Simultaneously, the associated acupoint's properties and polarity alter depending on the surrounding skin. Acupuncture points close to the damaged area are typical; however, they are not always positioned far away from the damaged area. The concept of semi-conduction is essential to the knowledge of acupuncture's mechanism.

Albert Szent Gorgyi, a pioneer of current concepts, offers ionic conduction to conduct energy via charged particles (ions) [16]. It is how nerve impulses are transmitted out. A membrane that protects nerve fibers, for example, is an example of an ionic current that works well over short distances but breaks down over longer distances. Semiconduction creates and induces a current by allowing electrons to move from one nucleus through a highly ordered material structure. If the temperature is suitable, semiconductors can act as conductors or as insulators. They can only transmit low currents, yet the current can travel over great distances quickly. This notion can be used in various traditional electric circuits, the diode gate's most well-known. The semi-conductor prevents the free charge flow in the event of injury, resulting in charge accumulation and pain [17]. The core explanation of how acupuncture works is around the concept of a diode, which is a semi-conductor that only enables current to flow in one direction [15]. The pictures below (Fig. 1) demonstrate this:

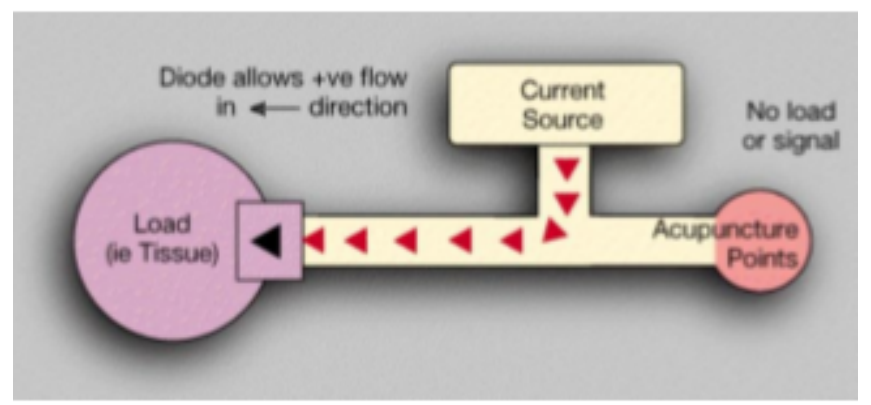

Fig. 1. Healthy tissue.

As of right now, the only source of current is the body's metabolism. The load is the part of the body that is connected to a specific acupoint on the diagram. If the load or tissue is normal, the acupuncture spot does not show change. Fig. 2 shows a current of injury when the load is damaged. The flow of current into that site is obstructed. Acupoints are activated as a result of the current flowing back from the origin of the current.

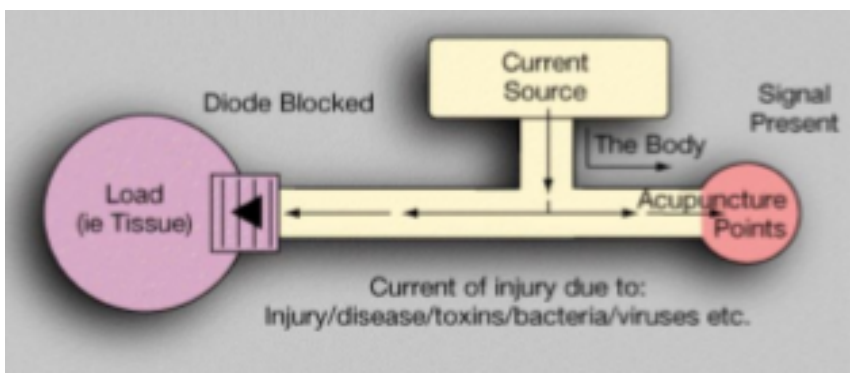

Fig. 2. In case of injury.

Fig. 3 shows the effect of grounding with the treatment of acupuncture.

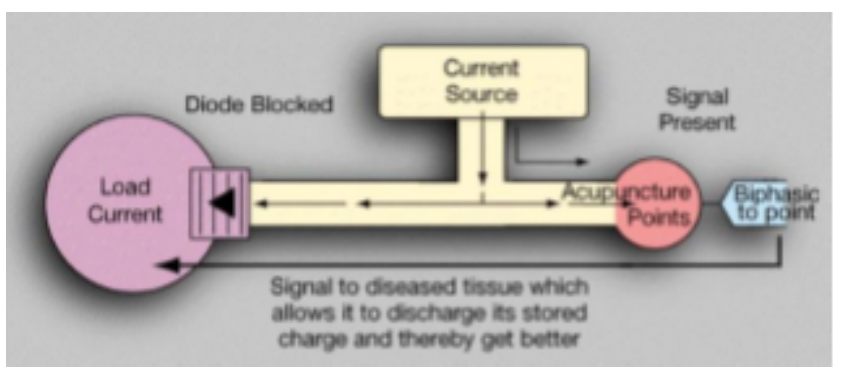

Fig. 3. Grounding with acupuncture.

The skin around acupuncture points is often negatively charged. Higher negative values represent increased electrical activity in the associated anatomical location. It's found in functional pathology when there's no physical harm or organic alteration. There's a correlation between this level and severe pain in acute conditions [15]. When a bipolar electric current is passed through semi-conducting skin, the typical conductive qualities are restored, the stored charges are 
released from the injured area, and the disease signs are reduced. In some cases, the problem can be eliminated with the right amount of therapy.

According to current research, the grounding of the human body has a significant effect on bioelectrical and bioenergetic processes and a significant moderating effect on chronic disorders and dysfunction [18]. Moreover, Oschman and colleagues postulated that when the body is connected to the Earth, free electrons from the Earth's surface are transferred to the body, where they'll have antioxidant effects [19]. It is necessary to conduct further research to validate these theories.

\section{WHAT IS ACUPUNCTURE WITH GROUNDING?}

Grounding with acupuncture is a modern variation of acupuncture. It uses electricity to enhance the benefits of this traditional treatment. Like traditional acupuncture, electroacupuncture uses needles placed in the same spots. Then, a small electrode is attached to the needles. A small amount of electricity runs through the electrode and gives a slight vibration or soft hum during treatment.

The electrode gives off the effect of your acupuncturist activating the acupuncture point with their hands. It ensures you get the right amount of stimulation during your whole treatment. The treatments are also shorter because the electrodes can activate your acupuncture points more quickly. A benefit of using electroacupuncture is that it can cover larger areas of your body than traditional acupuncture. This ability makes this type of acupuncture more accurate than others.

\section{A. How Does Electroacupuncture Work?}

Electroacupuncture uses needles in the meridian point, acupuncture point, and then connects to the Earth or earthing source that passes a small electric current through the needle.

When the body is grounded, free-electron flow to or from the body occurs, resulting in a microcurrent in the body. The gate control theory best explains how this microcurrent aids in the healing of various processes. The best justification for using high-intensity milliampere stimulation is the Melzack and Wall [20] gate control theory. According to Melzack and Wall [20], high-intensity current activation restricts gates to associated nerve transmissions. In the substantial gelatinosa, activation of the beta-sensory neurons, which react faster than the shorter pain-transmitting C-fibers, would tend to block pain signals from reaching the spinal cord. The beta sensory input floods the circuits, effectively blocking the pain signals passing from the $\mathrm{C}$-fibers. In the central nervous system, endorphins and enkephalins, the body's naturally occurring narcotics, also increase the secretion for this presynaptic feedback inhibition. The tissues would be irritated sufficiently by high-intensity electrical stimulation to activate the endogenous opiates' defensive reaction. The pain relief will persist for several hours. Unfortunately, such benefits mask the pain for a short time, with no long-term cumulative effects and no significant effects on the body's healing process.

On the other hand, microcurrent stimulation is thought to be a technique to start the body's natural healing mechanism via the electro-physiological system, rather than suppressing the pain of disease [20]. The benefits of microcurrent are said to be the cumulative pain-relieving effects. The next-day carry-over is well-known among microcurrent users. Patients may not experience instant analgesia, but they report significant subjective improvement the next day, backed up by clinical assessment, which shows reduced pain with palpation, reduced inflammation, normalized skin coloration, and increased mobility range [21].

The main points of the mechanism are:

1. Grounding acupuncture also helps to decrease your pain by activating bioactive chemicals in your body. This process may decrease your sensitivity to the pain you're feeling. It's also been shown to help reduce inflammation in your body.

2. When you get electroacupuncture, it activates your sympathetic nerve fibers. By activating these fibers, your body releases endogenous opioids such as endorphins, which help to reduce inflammation. The activated nerve fibers may also help to reduce feelings of persistent pain. It makes it a good treatment for chronic pain and stress.

3. Studies have also found that electroacupuncture may help your body release mesenchymal stem cells (MSCs) into your bloodstream. MSCs are adult stem cells primarily found in your bone marrow. MSCs help your body create different types of tissues. They also have healing properties.

4. Electroacupuncture is believed to help increase your blood flow. It may help to relieve any pain you're feeling. In addition, this type of acupuncture warms your muscles. It may help with poor circulation and potential blockages.

5. Another condition that electroacupuncture has been shown to reduce is chronic stress. Studies show that this type of therapy can help you reduce long-term chronic stress through stimulation.

For many centuries, acupuncture has been used to treat pain, including chronic pain disorders. Electroacupuncture is a more modern form of acupuncture, and it has become a substitute for traditional acupuncture.

\section{DISCUSSION}

Acupuncture's concept has been proven through numerous investigations. On the other hand, most acupuncture research has concentrated on the efficacy of acupuncture instead of the acupuncture process or stimulation mechanism, both of which might alter acupuncture's effectiveness. Progress in exploring the principle of acupuncture was made by Cho et al. [4] who used fMRI to detect brain reactions to acupuncture stimulation. Unfortunately, the study results were examined neurology instead of meridian theory, and electrical grounding was not considered.

Electric impulses produced in the human body are transmitted to all parts, causing electric interference before developing electric potentials at particular body parts. Similarly, in terms of grounding, the principle of acupuncture might be described by the interaction of bioelectrical charges.

Electroacupuncture is somewhat new, so there is little proof that it can be beneficial for many uses. Despite this, some research indicates that it may help with chemotherapy side effects, acute pain, and arthritis. 


\section{A. Arthritis}

A 2005 review looked at two studies exploring the benefits of acupuncture for rheumatoid arthritis (RA) [22]. One study used acupuncture with grounding treatments. This treatment resulted in a significant improvement in knee pain just 24 hours following treatment in one trial. This effect can remain for up to four months following treatment. However, the review authors note that the study included only a small number of participants and was of low quality.

A more recent literature review from 2017 looked at 11 randomized controlled trials on grounding acupuncture for knee osteoarthritis [23]. The results suggest that it helped to both reduce pain and improve movement. The authors noted that the studies seemed to suggest four weeks of treatment were needed. The study authors concluded by emphasizing the need for more high-quality trials to support grounding with acupuncture's treatment benefits.

\section{B. Acute pain}

A 2014 literature review looked at multiple preclinical animal studies on grounding with acupuncture's use as a form of pain relief [24]. The results suggest that it can help to reduce different types of pain. The authors also found evidence that a combination of grounding acupuncture and pain medication may be more effective than medication alone. It is promising, as it could mean that using grounding for pain relief may reduce the need for high doses of medicine. Keep in mind that these results came from animal studies. More research is needed to understand the effects of this therapy on pain in humans.

\section{Chemotherapy-related Nausea}

A 2005 review of 11 randomized trials looked at acupuncture to reduce chemotherapy-related vomiting [25]. The authors noted that acupuncture stimulation appeared to be more helpful for reducing vomiting right after a chemotherapy treatment than standard acupuncture.

\section{CONCLUSION}

Acupuncture is a well-studied and evidence-based practice that has been used successfully for thousands of years. We do need more research on adding grounding to this ancient practice. Grounding acupuncture is closely related to acupuncture, but it involves stimulating needles with an electrical current provided by earthing. Some believe that this micro current enhances the healing properties of traditional acupuncture.

The decision to use grounding in treatment is characterized by various considerations, including the ailment being treated, the intensity of symptoms, individual patient characteristics, and the choice and expertise of the practitioners. It is essential to understand what medical decisions should be guided (for example, when to include grounding and the specific stimulation parameters that should be used to find out what appears best for certain patients and certain conditions and assess the experiences of patients with grounding compared with traditional acupuncture). Finally, criteria based on practitioners' and patients' clinical experience, as gathered through well-designed interviews or surveys, should be devised and implemented to design clinical studies comparing grounding with traditional acupuncture. There's limited evidence to support the many claims made about grounding with acupuncture. But the research that does exist suggests it may help with several health issues, including arthritis, acute pain, and chemotherapy side effects.

\section{REFERENCES}

[1] G. A. Ulett, J. Han, and S. Han. "Traditional and evidence-based acupuncture." J uth Med J, 91(12): 115, 1998.

[2] Research Group of Acupuncture Anesthesia, Peking Medical College. "The role of some neurotransmitters of brain in finger-acupuncture analgesia." Scientia Sinica, 17: 112-130, 1974.

[3] K.Wang and J. Liu. "Needling sensation receptor of an acupoint supplied by the median nerve-studies of their electro-physiological characteristics." The American journal of Chinese medicine, 17.03n04:145-155, 1989.

[4] Z. H. Cho, Y. M. Ro and T. H. Lim. "NMR venography using the susceptibility effect produced by deoxyhemoglobin." Magnetic resonance in medicine, 28(1): 25-38, 1992.

[5] M. W. Ho, "Quantum coherence and conscious experience." Kybernetes, 1997.

[6] R. O. Becker, "Exploring new horizons in electromedicine." 17-18, 2004.

[7] W. A. Tiller, "What do electrodermal diagnostic acupuncture instruments really measure." American Journal of Acupuncture 15(1): 15-23, 1987.

[8] H. Motoyama, "Electro-physiological and preliminary biochemical studies of skin properties in relation to the acupuncture meridian." International Association for Religion and Parapsychology 6: 1-36, 1980 .

[9] A. Ogata, J. Sugenoya, N. Nishimura, and T. Matsumoto, "Low and high frequency acupuncture stimulation inhibits mental stress-induced sweating in humans via different mechanisms," Autonomic Neuroscience 118(1-2): 93-101, 2005.

[10] T. Matsumoto, F. M. Jr. "Acupuncture, electric phenomenon of the skin, and postvagotomy gastrointestinal atony." The American Journal of Surgery 125(2): 176-180, 1973.

[11] Y. H. Lee, Q. J. Lee, E. G. Kim, H. S. Kim, T. M. Shin, "Influence on meridians electric potential for electrical ground and insulation," Journal of the Korea Institute of Information and Communication Engineering, 10(12): 2243-2250, 2006.

[12] P. Madill, "The Uses and Limitations of Acupuncture Point Measurement, German Electroacupuncture or Electroacupuncture According to Voll (EAV)," American Journal of Acupuncture 12(1):33-42, 1984.

[13] K. G. Chen, "Electrical properties of meridians," IEEE Engineering in Medicine and Biology Magazine 15(3):58-63, 1996.

[14] H. Tan, S. Tumilty, C. Chapple, L. Liu, S. McDonough, H. Yin... G. D. Baxter, "Understanding acupoint sensitization: a narrative review on phenomena, potential mechanism, and clinical application," EvidenceBased Complementary and Alternative Medicine 2019;2019.

[15] G. A. Ulett, J. Han, S. Han, "Traditional and evidence-based acupuncture," South Med J. 91:1115-20, 1998.

[16] Y. Omura, "Electro-acupuncture: its electro-physiological basis and criteria for effectiveness and safety-part I," Acupuncture \& ElectroTherapeutics Research 1(1-2):157-81, 1975.

[17] R. Pethig, A. Szent-Györgyi, "Bioelectrochemistry, the living state, and electronic conduction in proteins," Bioelectrochemistry: Springer; 227-52, 1980.

[18] K. Sokal, P. Sokal, "Earthing the human body influences physiologic processes," The Journal of Alternative and Complementary Medicine 17(4):301-8, 2011.

[19] J. L. Oschman, G. Chevalier, R. Brown, "The effects of grounding (earthing) on inflammation, the immune response, wound healing, and prevention and treatment of chronic inflammatory and autoimmune diseases," Journal of Inflammation Research 8:83, 2015.

[20] R. Melzack, J. Katz, "The gate control theory: Reaching for the brain," Pain: psychological perspectives 13-3, 2004.

[21] R. I. Picker, "Low-volt pulsed microamp stimulation," Part II Clinical Management 9:28-33, 1989.

[22] L. Casimiro, L. Barnsley, L. Brosseau, S. Milne, V. Welch, P. Tugwell and G. A. Wells, "Acupuncture and electroacupuncture for the treatment of rheumatoid arthritis," Cochrane Database of Systematic Reviews, (4), 2005. 
[23] N. Chen, J. Wang, A. Mucelli, X. Zhang, and C. Wang, "Electroacupuncture is beneficial for knee osteoarthritis: the evidence from meta-analysis of randomized controlled trials," The American journal of Chinese medicine, 45(05): 965-985, 2017.

[24] R. Zhang, L. Lao, K. Ren, B. M. Berman, "Mechanisms of acupuncture-electroacupuncture on persistent pain," Anesthesiology 120(2):482-503, 2014

[25] J. Ezzo, A. Vickers, M. A. Richardson, C. Allen, S. L. Dibble, B. Issell, L. Lao, M. Pearl, G. Ramirez, J. A. Roscoe, J. Shen, J. Shivnan, K. Streitberger, I. Treish, G. Zhang, "Acupuncture-point stimulation for chemotherapy-induced nausea and vomiting," $J$ Clin Oncol. 23(28):7188-98, 2005.

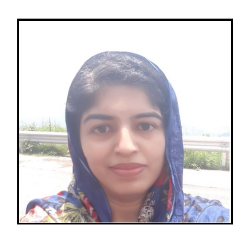

\section{Madiha Khalid}

Place of birth: Lahore.

Date of Birth: 11/9/1985.

M.Phil clinical Pharmacy.

Humdard University, Lahore.

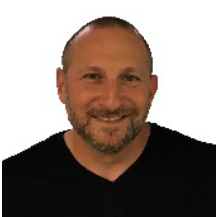

Jeremy Madvin

Place of Birth: Los Angeles, USA

Bate of Birth: 11/22/1967.

MBA International Business.

University of Redlands, United States. 